University of Wollongong

Research Online

Faculty of Engineering and Information

Faculty of Engineering and Information

Sciences - Papers: Part A

Sciences

2003

Chain entanglements and fracture energy in interfaces between immiscible polymers

Leonardo Silvestri

Scuola Normale Superiore di Pisa

Hugh Ralph Brown

University of Wollongong, hbrown@uow.edu.au

Stefano Carra

MAPEI S.p.A.

Sergio Carra

Politecnico di Milano

Follow this and additional works at: https://ro.uow.edu.au/eispapers

Part of the Engineering Commons, and the Science and Technology Studies Commons

Research Online is the open access institutional repository for the University of Wollongong. For further information contact the UOW Library: research-pubs@uow.edu.au 


\title{
Chain entanglements and fracture energy in interfaces between immiscible polymers
}

\begin{abstract}
It is a very well-known experimental fact that the toughness of interfaces obtained by joining pairs of immiscible glassy polymers is strongly correlated to the interfacial width. Several models have been proposed in the literature to estimate the fracture energy of these interfaces, but the agreement displayed with the experimental data cannot be considered satisfactory. In this paper a new model is proposed for polymers with molecular weight higher than the critical value for the onset of entanglements. The model is based on a precise and realistic calculation of the areal density of entangled strands across the interface, that is the crucial parameter determining the toughness of the glassy joints. In this paper a new fracture regime is also introduced, called "partial crazing," corresponding to a situation where, due to the fact that some of the load-bearing strands are broken during plastic deformation, the craze can start, but not fully develop. Model predictions are then compared with a series of literature fracture energy experimental data, showing excellent agreement.
\end{abstract}

\section{Keywords}

immiscible, between, polymers, interfaces, chain, energy, fracture, entanglements

Disciplines

Engineering | Science and Technology Studies

\section{Publication Details}

Silvestri, L., Brown, H. R., Carra, S. \& Carra, S. (2003). Chain entanglements and fracture energy in interfaces between immiscible polymers. Journal of Chemical Physics, 119 (15), 8140-8149. 


\section{AD $\mid \begin{aligned} & \text { The Journal of } \\ & \text { Chemical Physics }\end{aligned}$}

Chain entanglements and fracture energy in interfaces between immiscible polymers

Leonardo Silvestri, Hugh R. Brown, Stefano Carrà, and Sergio Carrà

Citation: The Journal of Chemical Physics 119, 8140 (2003); doi: 10.1063/1.1610444

View online: http://dx.doi.org/10.1063/1.1610444

View Table of Contents: http://scitation.aip.org/content/aip/journal/jcp/119/15?ver=pdfcov

Published by the AIP Publishing

\section{Articles you may be interested in}

Nanoscale plastic deformation and fracture of polymers studied by in situ nanoindentation in a transmission electron microscope

Appl. Phys. Lett. 88, 181908 (2006); 10.1063/1.2200718

Investigations on several empirical rules for entangled polymers based on a self-consistent full-chain reptation theory

J. Chem. Phys. 112, 8176 (2000); 10.1063/1.481418

Effect of pressure on the segmental and chain dynamics of polyisoprene. Molecular weight dependence J. Chem. Phys. 111, 9847 (1999); 10.1063/1.480327

Semi-flexible polymers at a liquid-liquid interface: Self-consistent field calculations

J. Chem. Phys. 109, 4592 (1998); 10.1063/1.477064

Explanation for SlipStick Melt Fracture in Terms of Molecular Dynamics in Polymer Melts

J. Rheol. 29, 605 (1985); 10.1122/1.549804

\section{AlP hameded}

Journal of Applied Physics is pleased to announce André Anders as its new Editor-in-Chief 


\title{
Chain entanglements and fracture energy in interfaces between immiscible polymers
}

\author{
Leonardo Silvestria) \\ Scuola Normale Superiore, Piazza dei Cavalieri 7, 56126 Pisa, Italy \\ Hugh R. Brown \\ BHP Institute of Steel Processing and Products, University of Wollongong, \\ Wollongong NSW 2522, Australia \\ Stefano Carrà \\ MAPEI S.p.A., via Cafiero 22, 20158 Milano, Italy \\ Sergio Carrà \\ Dipartimento di Chimica, Materiali e Ingegneria Chimica "G. Natta," Politecnico di Milano, \\ via Mancinelli 7, 20131 Milano, Italy
}

(Received 29 May 2003; accepted 25 July 2003)

\begin{abstract}
It is a very well-known experimental fact that the toughness of interfaces obtained by joining pairs of immiscible glassy polymers is strongly correlated to the interfacial width. Several models have been proposed in the literature to estimate the fracture energy of these interfaces, but the agreement displayed with the experimental data cannot be considered satisfactory. In this paper a new model is proposed for polymers with molecular weight higher than the critical value for the onset of entanglements. The model is based on a precise and realistic calculation of the areal density of entangled strands across the interface, that is the crucial parameter determining the toughness of the glassy joints. In this paper a new fracture regime is also introduced, called "partial crazing," corresponding to a situation where, due to the fact that some of the load-bearing strands are broken during plastic deformation, the craze can start, but not fully develop. Model predictions are then compared with a series of literature fracture energy experimental data, showing excellent agreement.

(C) 2003 American Institute of Physics. [DOI: 10.1063/1.1610444]
\end{abstract}

\section{INTRODUCTION}

Adhesion between pairs of immiscible polymers is a phenomenon of great importance in many practical applications, as it determines the mechanical properties of polymer mixtures often used to make, for example, composites, adhesives, and coextruded materials. ${ }^{1}$ Bulk glassy polymers are tough materials, the source of toughness being entanglements. Similarly, the strength of an interface between two immiscible glassy polymers is determined by the density of the entanglements that cross it. Experimental work, usually performed using a cantilever beam test, has shown that, for example, interfacial fracture energy between polystyrene (PS) and polymethylmethacrylate (PMMA) has a value of around $12 \mathrm{~J} / \mathrm{m}^{2}, 2,3$ substantially greater than the ideal work of adhesion, but much less than bulk fracture energy of both polymers. More recently, Schnell et al. ${ }^{4}$ used bilayers of PS and polyparamethylstyrene (PpMS), to obtain a wide range of interfacial widths by changing the annealing temperature of the samples. By doing so, they were able to demonstrate that there is a clear correlation between the width, measured by neutron reflectivity, and the fracture energy of the interface. The results were confirmed in another work by the same authors on interfaces between PS and the statistical

a)Electronic mail: 1.silvestri@sns.it copolymer of poly(bromostyrene-styrene) $\left(\mathrm{PBr}_{x} \mathrm{~S}\right) .{ }^{5}$ The same correlation has been found by Brown, ${ }^{6}$ who measured the toughness of the interface between a random copolymer P(S-r-PMMA) and pure PMMA, for different fractions of PS in the copolymer.

The importance of entanglements has been proved by Creton et al. ${ }^{7}$ where an interface between PS and poly 2-vinylpiridine (PVP) was reinforced with block copolymers of PS and PVP. Particularly, in tests in which molecular masses were varied, the results showed that for short blocks, that are not long enough to be entangled with their homopolymers, only small increases of fracture energy can be obtained. Chain coupling across the interface can be described by the areal density of effectively entangled strands, indicated by $\Sigma_{\text {eff }}$. A strand is said to be effectively entangled if it connects two subsequent entanglements placed on different sides of the interface, and is therefore able to transfer stress across the interface, as shown in Figs. 1(a) and 1(b). Again, block copolymer reinforced interfaces, supplemented by surface analysis to determine on which side the block copolymer ends up after failure, showed that different fracture regimes can occur depending on the value of $\Sigma_{\text {eff }} .8$

It is therefore clear that, in any model that aims to describe fracture of entangled polymers, $\Sigma_{\text {eff }}$ will play a key role, since it is proportional to the maximum stress an interface can withstand. For symmetric interfaces a simple model 
a)

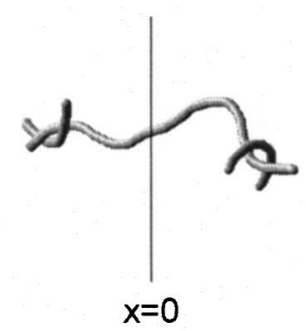

b)

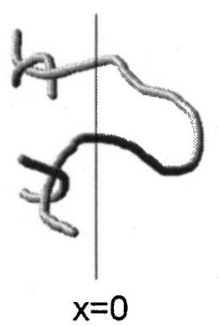

c)

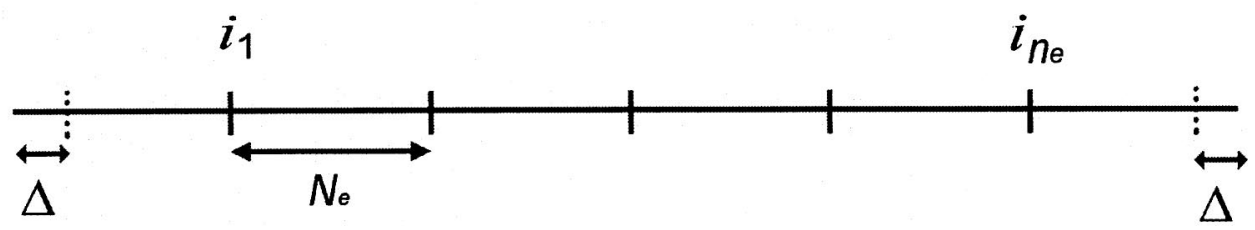

FIG. 1. Schematic representation of an effective (a) and a not effective (b) entanglement; the planar interface is defined by $x=0$. (c) Position of the $n$ e entanglements along the chain. Continuous bars represent entangled chains crossings, while dashed ones are just an aid to the eye. Distances are expressed in number of monomer units.

similar to Lake and Thomas theory of elastomers, would predict $\Sigma_{\text {eff }}=\rho_{e} L_{e} / 2$, where $\rho_{e}$ is the density of entanglements and $L_{e}$ is the root-mean-square end to end distance between them. ${ }^{9}$ A more accurate expression was derived by Mikos and Peppas, ${ }^{10}$ who used a stochastic approach to count the number of coupling strands across a fracture plane in bulk polymers and included chain end effects in their analysis.

For weakly immiscible polymer pairs, de Gennes ${ }^{11}$ proposed a scaling law for the dependence of $\Sigma_{\text {eff }}$ on the interface width through the Flory-Huggins interaction parameter $\chi$. However, his result is based on energetic considerations, and it does not take into account the effect of inhomogeneous polymer densities at the interface.

In recent years a new approach has been proposed by Brown $^{6}$ to relate the interfacial width of an interface to its toughness, in which $\Sigma_{\text {eff }}$ depends only on the concentration profile of the polymer. In particular, Brown neglected chain end effects and assumed that the probability that a strand starting from $x$ might end in $x^{\prime}$ is proportional to the ratio of the polymer volume fractions at the two points, obtaining the density of effective entanglements formed by one species as

$$
\Sigma_{\mathrm{eff}}=2 \rho_{e} \int_{-L_{e}}^{0} d x \phi(x) \int_{0}^{L_{e}+x} d x^{\prime} \frac{\phi\left(x^{\prime}\right)}{2 L_{e} \phi(x)}
$$

which in the homogeneous case, $\phi \equiv 1$, gives $\Sigma_{\text {eff }}=\rho_{e} L_{e} / 2$, as predicted by the simple model resembling Lake and Thomas theory. As discussed in Appendix B the probability distribution is in fact roughly proportional to the square root of the volume fractions ratio; as a result, the equation derived by Brown predicts a variation of the density of effective entangled chains that is too slow with respect to the changes in the interface width.

In the present work we propose a rigorous model for the calculation of $\Sigma_{\text {eff }}$, avoiding most of the simplifications affecting previous works. The paper is organized as follows. In Sec. II we derive an explicit expression to compute $\Sigma_{\text {eff }}$, using the Green function formalism in the context of a mean- field approach. In Sec. III we describe the fracture mechanisms of interest for an entangled interface and introduce a new intermediate regime called partial crazing; numerical results are discussed in Sec. IV and compared to available experimental data. Conclusions are finally presented in Sec. V. Appendixes A and B are devoted to two aspects of the method proposed in Sec. II to calculate $\Sigma_{\text {eff }}$ : in the first we present a long-chain approximation and in the second we describe our method using a stochastic language.

\section{CALCULATION OF THE EFFECTIVE ENTANGLEMENT DENSITY FOR ASYMMETRIC INTERFACES}

As described in the Introduction, experiments have demonstrated the importance of entanglements in determining the toughness of interfaces. The crucial point of our fracture model is therefore the calculation of $\Sigma_{\text {eff }}$. In an asymmetric interface it can be written as the sum of the effective entanglements formed by the two polymers

$$
\Sigma_{\mathrm{eff}}=\Sigma_{\mathrm{eff}}^{(A)}+\Sigma_{\mathrm{eff}}^{(B)}
$$

Calculations will be carried out for polymer A, and we will always refer to this species, unless otherwise stated. Derivations would obviously be the same for polymer B.

We assume that the two polymers are in thermodynamic equilibrium at an annealing temperature above their $T_{g}$, and we will use, in our derivation, a mean-field approximation that is suitable for melts. Each polymer chain can therefore be viewed as an ideal Gaussian chain, submitted to the mean external field created by all the other chains. Consider a polymer chain of molecular weight $M$, made of $N$ repeating units of molecular weight $M_{0}$. In order to account for chain stiffness, in our derivation we will take into consideration the 
equivalent Gaussian chain of the actual macromolecule. Statistical units of the equivalent chain have molecular weight $M_{0}$ and length $b$, given by

$$
b=l \sqrt{C_{\infty} j},
$$

where $j$ is the number of backbone bonds of the original repeating unit, $C_{\infty}$ is the chain stiffness, and $l$ the bond length, which is $1.54 \AA$ for $\mathrm{C}-\mathrm{C}$ bonds.

The equivalent Gaussian chain can be subdivided into segments of molecular weight $M_{e}$, containing $N_{e}=M_{e} / M_{0}$ monomers, and, following Mikos and Peppas, ${ }^{10}$ we assume that an entanglement is found at the end of each segment excluding the final one. A chain with molecular weight $M$ therefore has $n=M / M_{e}$ segments and $n-1$ entanglements. Due to the mean-field approximation, in our derivation we will solve a single chain problem, and, to perform the calculations, it is necessary to assume that the exact position of the entanglements along the chain is known. We will therefore consider an integer number of entanglements $n_{e}=[n]$ -1 , where $[x]$ denotes the integer part of $x$. Moreover, since the two ends of a chain are completely equivalent, it can be safely assumed that, on the average, entanglements are symmetrically distributed with respect to the chain center. Entanglements are therefore located at positions $i_{k}=k N_{e}+\Delta$ along the chain, where $\Delta=\left(N-N_{e}[n]\right) / 2$ and $k=1, \ldots, n_{e}$, as shown in Fig. 1(c). Rigorously, every physical quantity that depends on the entanglement positions should be obtained as a mean over all the possible configurations $\left\{i_{k}\right\}$, but for the moment we treat the entanglement as fixed along the chain. The validity of this assumption will be discussed in Sec. IV. Another hypothesis assumed in our derivation is that the molecular weight of entanglement stays constant throughout the whole interface; this approximation will also be discussed.

In order to solve the problem we introduce the Green function formalism. At thermodynamic equilibrium, the Green function $G\left(\mathbf{r}, \mathbf{r}^{\prime} ; N\right)$ represents the statistical weight of chains starting at $\mathbf{r}$ and ending at $\mathbf{r}^{\prime}$ in $N$ steps, normalized with respect to the value it assumes in the absence of external fields.

The Green function of a Gaussian chain in the presence of an external field $U_{e}$ satisfies the following differential equation: $^{12}$

$$
\begin{gathered}
\left(\frac{\partial}{\partial N}-\frac{b^{2}}{6} \frac{\partial^{2}}{\partial \mathbf{r}^{2}}+\frac{1}{k_{B} T} U_{e}(\mathbf{r})\right) G\left(\mathbf{r}^{\prime}, \mathbf{r} ; N\right) \\
=\delta\left(\mathbf{r}-\mathbf{r}^{\prime}\right) \delta(N),
\end{gathered}
$$

where the right-hand side term is set so that it can satisfy the proper boundary conditions. For $U_{e}=0$, Green function gives the well-known Gaussian distribution function

$$
G_{0}\left(\mathbf{r}, \mathbf{r}^{\prime} ; N\right)=\left(\frac{2 \pi N b^{2}}{3}\right)^{-3 / 2} \exp \left(-\frac{3\left(\mathbf{r}-\mathbf{r}^{\prime}\right)^{2}}{2 N b^{2}}\right)
$$

The mean values of any physical quantity depending on the position $\mathbf{r}_{n}$ of the $n$th monomer are given by ${ }^{12}$

$$
\left\langle A\left(\mathbf{r}_{n}\right)\right\rangle=\frac{\int d \mathbf{r}_{0} d \mathbf{r}_{n} d \mathbf{r}_{N} G\left(\mathbf{r}_{0}, \mathbf{r}_{n} ; n\right) G\left(\mathbf{r}_{n}, \mathbf{r}_{N} ; N-n\right) A\left(\mathbf{r}_{n}\right)}{\int d \mathbf{r}_{0} d \mathbf{r}_{N} G\left(\mathbf{r}_{0}, \mathbf{r}_{N} ; N\right)} .
$$

If a quantity depends on the position of two monomers, the corresponding expression for its mean value is

$$
\left\langle A\left(\mathbf{r}_{n}, \mathbf{r}_{m}\right)\right\rangle=\frac{\int d \mathbf{r}_{0} d \mathbf{r}_{n} d \mathbf{r}_{m} d \mathbf{r}_{N} G\left(\mathbf{r}_{0}, \mathbf{r}_{n} ; n\right) G\left(\mathbf{r}_{n}, \mathbf{r}_{m} ; m-n\right) G\left(\mathbf{r}_{m}, \mathbf{r}_{N} ; N-m\right) A\left(\mathbf{r}_{n}, \mathbf{r}_{m}\right)}{\int d \mathbf{r}_{0} d \mathbf{r}_{N} G\left(\mathbf{r}_{0}, \mathbf{r}_{N} ; N\right)},
$$

with $m>n .^{12}$

Since in the case of a plane interface the external potential depends only on one coordinate, that we identify as $x$, we can integrate on the other two and work in one dimension. The Green function $G_{0}$ becomes

$$
G_{0}\left(x, x^{\prime} ; N\right)=\frac{\beta_{N}}{\sqrt{\pi}} \exp \left[-\beta_{N}^{2}\left(x-x^{\prime}\right)^{2}\right]
$$

with

$$
\beta_{N}=\frac{\sqrt{3 / 2}}{N^{1 / 2} b}
$$

To compute $\Sigma_{\text {eff }}$ it is necessary to count every strand that connects two subsequent entanglements placed on different sides of the interface, that we assume to be at $x=0$. Working in a mean-field approximation, it is sufficient to consider the single chain problem and then multiply the result by the number of chains. The operator that counts the total number of the coupling strands across the interface per unit area is

$$
\begin{aligned}
\hat{\Sigma}_{\mathrm{eff}}= & \frac{\nu}{A} \sum_{k=1}^{n_{e}-1}\left[\int_{x<0} d \mathbf{r} \int_{x>0} d \mathbf{r}^{\prime} \delta\left(\mathbf{r}_{i_{k}}-\mathbf{r}\right) \delta\left(\mathbf{r}_{i_{k+1}}-\mathbf{r}^{\prime}\right)\right. \\
& \left.+\int_{x>0} d \mathbf{r} \int_{x<0} d \mathbf{r}^{\prime} \delta\left(\mathbf{r}_{i_{k}}-\mathbf{r}\right) \delta\left(\mathbf{r}_{i_{k+1}}-\mathbf{r}^{\prime}\right)\right]
\end{aligned}
$$

where the sum over $k$ counts entanglements along the chain, $A$ is the system area, and $\nu$ is the total number of chains. The density of effective entanglements per unit area can be expressed as the mean value of the above-defined operator

$$
\Sigma_{\text {eff }}=\left\langle\hat{\Sigma}_{\text {eff }}\right\rangle
$$

From Eq. (7) we then obtain 


$$
\begin{aligned}
\Sigma_{\mathrm{eff}}= & \frac{\nu}{A} \frac{1}{\iint d \mathbf{r}_{0} d \mathbf{r}_{N} G\left(\mathbf{r}_{0}, \mathbf{r}_{N} ; N\right)} \\
& \times \sum_{k=1}^{n_{e}-1}\left[\int_{x<0} d \mathbf{r} \int_{x>0} d \mathbf{r}^{\prime} \iint d \mathbf{r}_{0} d \mathbf{r}_{N}\right. \\
& \times G\left(\mathbf{r}_{0}, \mathbf{r} ; i_{k}\right) G\left(\mathbf{r}, \mathbf{r}^{\prime} ; N_{e}\right) G\left(\mathbf{r}^{\prime}, \mathbf{r}_{N} ; N-i_{k+1}\right) \\
& +\int_{x<0} d \mathbf{r}^{\prime} \int_{x>0} d \mathbf{r} \iint d \mathbf{r}_{0} d \mathbf{r}_{N} G\left(\mathbf{r}_{0}, \mathbf{r} ; i_{k}\right) \\
& \left.\times G\left(\mathbf{r}, \mathbf{r}^{\prime} ; N_{e}\right) G\left(\mathbf{r}^{\prime}, \mathbf{r}_{N} ; N-i_{k+1}\right)\right]
\end{aligned}
$$

Integrating over $y$ and $z$ we obtain

$$
\begin{aligned}
\Sigma_{\mathrm{eff}}= & \frac{\nu}{A} \frac{1}{\int q(x ; N) d x} \\
& \times \sum_{k=1}^{n_{e}-1}\left[\int_{-\infty}^{0} d x \int_{0}^{\infty} d x^{\prime} q\left(x ; i_{k}\right) G\left(x, x^{\prime} ; N_{e}\right)\right. \\
& \times q\left(x^{\prime} ; N-i_{k+1}\right)+\int_{-\infty}^{0} d x^{\prime} \int_{0}^{\infty} d x q\left(x ; i_{k}\right) \\
& \left.\times G\left(x, x^{\prime} ; N_{e}\right) q\left(x^{\prime} ; N-i_{k+1}\right)\right]
\end{aligned}
$$

where the notation

$$
q(x ; n) \equiv \int G\left(x, x^{\prime} ; n\right) d x^{\prime},
$$

has been introduced.

Expression (13) can be simplified by noting that $G\left(x, x^{\prime} ; m\right)=G\left(x^{\prime}, x ; m\right)$ and that, since we assumed entanglements symmetrically distributed with respect to the chain center, $N-i_{k+1}=i_{n_{e}-k}$. Using these relationships it is possible to write

$$
\begin{aligned}
& \int_{-\infty}^{0} d x^{\prime} \int_{0}^{\infty} d x \sum_{k=1}^{n_{e}-1} q\left(x ; i_{k}\right) G\left(x, x^{\prime} ; N_{e}\right) q\left(x^{\prime} ; N-i_{k+1}\right) \\
& =\int_{-\infty}^{0} d x^{\prime} \int_{0}^{\infty} d x \sum_{k=1}^{n_{e}-1} q\left(x^{\prime} ; i_{k}\right) G\left(x^{\prime}, x ; N_{e}\right) \\
& \quad \times q\left(x ; N-i_{k+1}\right)
\end{aligned}
$$

and finally

$$
\begin{aligned}
\Sigma_{\mathrm{eff}}= & \frac{\nu}{A} \frac{2}{\int_{L} q(x ; N) d x} \sum_{k=1}^{n_{e}-1}\left[\int_{-\infty}^{0} d x \int_{0}^{\infty} d x^{\prime} q\left(x ; i_{k}\right)\right. \\
& \left.\times G\left(x, x^{\prime} ; N_{e}\right) q\left(x^{\prime} ; N-i_{k+1}\right)\right]
\end{aligned}
$$

where $L$ is the dimension of the system in the $x$ direction. The total number of chains can be written as

$$
\nu=\frac{1}{N} \int_{V} \rho(\mathbf{r}) d \mathbf{r}=\frac{\rho_{b} A}{N} \int_{L} \phi(x) d x,
$$

where $\rho(\mathbf{r})$ is the total density of monomers, $\phi(x)$ $=[\rho(x)] / \rho_{b}$ is the polymer volume fraction, $\rho_{b}$ is the bulk monomer density, and $V$ is the system volume. Substituting Eq. (17) into Eq. (16), one derives

$$
\begin{aligned}
\Sigma_{\mathrm{eff}}= & \frac{2 \rho_{b}}{N} \frac{\int_{L} \phi(x) d x}{\int_{L} q(x ; N) d x} \sum_{k=1}^{n_{e}-1}\left[\int_{-\infty}^{0} d x \int_{0}^{\infty} d x^{\prime} q\left(x ; i_{k}\right)\right. \\
& \left.\times G\left(x, x^{\prime} ; N_{e}\right) q\left(x^{\prime} ; N-i_{k+1}\right)\right] .
\end{aligned}
$$

Finally, observe that in the homogeneous phases $\phi( \pm \infty)=q( \pm \infty ; N)$, as demonstrated in Appendix A, so that for a system where $L$ is much greater than the interface width we have

$$
\lim _{L \rightarrow \infty} \frac{\int_{L} \phi(x) d x}{\int_{L} q(x ; N) d x}=1 .
$$

The final expression for $\Sigma_{\text {eff }}$ is therefore

$$
\begin{aligned}
\Sigma_{\mathrm{eff}}= & \frac{2 \rho_{b}}{N} \sum_{k=1}^{n_{e}-1}\left[\int_{-\infty}^{0} d x \int_{0}^{\infty} d x^{\prime} q\left(x ; i_{k}\right)\right. \\
& \left.\times G\left(x, x^{\prime} ; N_{e}\right) q\left(x^{\prime} ; N-i_{k+1}\right)\right] .
\end{aligned}
$$

This equation gives the density of effective entanglements formed by one polymer across the interface. The total density is the sum of the contributions by both species. Expression (20) is the main result of this section.

In the case of an A/A interface, $U_{e}=0, \phi(x) \equiv 1$, $G\left(x, x^{\prime} ; N_{e}\right)=G_{0}\left(x, x^{\prime} ; N_{e}\right)$, and $q(x ; i) \equiv 1 \forall i$, so that from Eq. (20) it is

$$
\Sigma_{\mathrm{eff}}=\frac{2 \rho_{b}}{N}\left(n_{e}-1\right)\left[\int_{-\infty}^{0} d x \frac{1}{2}\left[1+\operatorname{Erf}\left(\beta_{N_{e}} x\right)\right] .\right.
$$

If the total number of entanglements is the real number $n_{e}=n-1$, then the final result in the symmetric case reads

$$
\Sigma_{\mathrm{eff}}=\frac{\rho_{b}(n-2)}{N \beta_{N_{e}} \sqrt{\bar{\pi}}} .
$$

That is exactly the same result that was obtained by Mikos and Peppas. ${ }^{10}$

\section{FRACTURE ENERGY}

In this section we describe how fracture energy of entangled interfaces can be calculated from $\Sigma_{\text {eff }}$.

As previously pointed out, here we are taking into consideration entangled chains, having $M>2 M_{e}$. For these molecular weights, it has been demonstrated by simulations that the force needed to disentangle a chain is higher than the breaking force of covalent $\mathrm{C}-\mathrm{C}$ bonds, so that the main microscopic failure mechanism is chain scission. ${ }^{13}$ At low entangled strands densities, $\Sigma_{\text {eff }}<\Sigma_{c}$, this is the only process dissipating energy, and therefore it is completely responsible for fracture energy. We can say that, in order to propagate the crack, chains entangled across the interface need to be broken. Following the classical Lake and Thomas approach, ${ }^{14}$ we assume that, when pulling a strand between two subse- 
quent entanglement, the supplied energy is shared between all bonds and, after breaking, it is dissipated. Under these assumptions the fracture energy is calculated as

$$
G_{c}^{(\mathrm{sc})}=U_{b}\left(\Sigma_{\text {eff }}^{(A)} N_{e}^{(A)} j^{(A)}+\Sigma_{\text {eff }}^{(B)} N_{e}^{(B)} j^{(B)}\right),
$$

where $U_{b}$ is the energy needed to break a $\mathrm{C}-\mathrm{C}$ bond, and all other quantities have already been defined.

If the interface is strong enough to sustain crazing stress $\sigma_{\text {craze }}$, then a plastic deformation occurs, capable of dissipating a huge amount of energy before the interface fails. The critical density for the onset of crazing is easily found as $\Sigma_{c}=\sigma_{\text {craze }} / f_{b}$, where $f_{b}$ is the maximum force that a $\mathrm{C}-\mathrm{C}$ bond can sustain. The crazing regime was described by Brown, ${ }^{15}$ who developed a model accounting for the stress transferred by cross-tie fibrils. This model was later refined by Sha and co-workers, ${ }^{16}$ who obtained the following expression for the fracture energy

$$
G_{c}^{(\mathrm{cr})}=\frac{\pi\left(1-v_{f}\right) \sigma_{\text {craze }} d}{-\alpha \log \left[1-\left(\sigma_{\text {craze }} / q \sum_{\text {eff }} f_{b}\right)^{2}\right]},
$$

where $v_{f}$ is the volume fraction of the fibrillated material in the craze, $d$ is the main fibril's spacing, and $\alpha$ is a dimensionless material constant, that depends on the effective Young modulus of the fibrils and on the angle between them. In Eq. (24) the effective density is corrected by a factor $q$ $<1$, defined as the fraction of strands that survive the craze formation. ${ }^{17}$ It follows that $G_{c}^{(\mathrm{cr})}$ is defined only if $q \Sigma_{\text {eff }}$ $>\Sigma_{c}$.

It is therefore advisable to introduce an intermediate regime, not yet taken into consideration in the literature, that could be called "partial crazing." In this regime $q \Sigma_{\text {eff }}<\Sigma_{c}$ $<\Sigma_{\text {eff }}$, meaning that the craze starts, but it cannot fully develop. What happens is that the plastic deformation takes place, but cross-tie fibrils are not yet created, so that lateral stress cannot be transferred. This partial crazing regime can be described as follows. When a craze develops, some of the load-bearing strands at the interface fail by chain scission. We assume that they are a constant fraction $w$ of the total broken strands, including also those in the craze but away from the interface. The work per unit area needed to create a craze of width $L$ is $\sigma_{\text {craze }} L\left(1-v_{f}\right)$. Assuming that the work is entirely spent to break entangled strands, which seems reasonable for the small craze widths we are considering, then the number of broken load-bearing strands per unit area is

$$
\Sigma_{\text {broken }}=w \frac{\sigma_{\text {craze }} L\left(1-v_{f}\right)}{U_{b} N_{e} j} .
$$

It is important to be careful in choosing $N_{e}$ and $j$. For large widths $L$ the majority of the broken strands probably belongs to the crazed material, while for small crazes there will be a consistent amount of strands of the other polymer. So, it would be correct to write $N_{e}$ and $j$ as functions of $L$, but as a first approximation it is reasonable to take values relative to the material in which the craze grows. The fraction of broken load-bearing strands is probably a function of $L$ too, but for the moment this complication will be neglected. The craze width can be calculated by imposing that its growth stops when the interface cannot sustain the crazing stress anymore, that is when $\Sigma_{\text {eff }}-\Sigma_{\text {broken }}=\Sigma_{c}$. From Eq. (25) the craze width is therefore

$$
L=\left(\Sigma_{\text {eff }}-\Sigma_{\mathrm{c}}\right) \frac{U_{b} N_{e} j}{w \sigma_{\text {craze }}\left(1-v_{f}\right)},
$$

and consequently

$$
G_{c}^{(\mathrm{pc})}=G_{c}^{(\mathrm{sc})}\left(\Sigma_{\mathrm{c}}\right)+\sigma_{\mathrm{craze}} L\left(1-v_{f}\right),
$$

where $G_{c}^{(\mathrm{sc})}\left(\Sigma_{c}\right)$ indicates the fracture energy calculated for the scission mechanism at the critical density. This term is added because when the $\Sigma_{\text {eff }}$ at the interface decreases to $\Sigma_{c}$ the interface fails by chain scission. The derived expression for the fracture energy in the regime of partial crazing is linear in $\Sigma_{\text {eff }}$ and is steeper than that obtained in the chain scission regime. The model also predicts that the critical width $L_{c}$, at which cross-tie fibrils start to transfer load and a craze can fully develop, is obtained for $\Sigma_{\text {eff }}=\Sigma_{c} / q$, and is therefore given by

$$
L_{c}=\left(\frac{1}{q}-1\right) \frac{U_{b} N_{e} j}{w f_{b}\left(1-v_{f}\right)} .
$$

\section{NUMERICAL RESULTS AND COMPARISON WITH LITERATURE EXPERIMENTAL DATA}

An accurate numerical evaluation of expression (20) has been obtained using the self-consistent mean-field (SCMF) calculations, combined with the modified diffusion equation (MDE) (4).

The approach can be briefly described. First we perform a standard SCMF calculation, following the method described in detail by Shull and co-workers, ${ }^{18}$ to obtain the interface width, probability functions $q(x ; i)$, and polymer mean fields. Then mean, fields can be inserted in the MDE, which is solved numerically to obtain the needed Green functions. Finally, expression (20) is calculated and the result for $\Sigma_{\text {eff }}$ is multiplied by $(n-2) /([n]-2)$ in order to obtain a smoother dependence on the molecular weight and to take into account the fact that real polymers are not monodisperse. Numerical results showed that rigidly shifting entanglement positions along the chain has no appreciable effects on the calculated $\Sigma_{\text {eff }}$, so that an average over all the possible positions of the first entanglement is not necessary.

In order to show the general dependence of $\Sigma_{\text {eff }}$ on the interfacial width, we applied the method to a simple illustrative system, simulating an interface between two materials with bulk parameters equal to those of PS and the same molecular weight $300 k$; the interfacial width is then changed by varying the interaction parameters $\chi$ from 0 to 0.05 . The calculated values of $\Sigma_{\text {eff }}$, obtained for this system with Eq. (20), are showed in Fig. 2 by joined full circles. For comparison, in the same figure we also show the results obtained by applying Brown's equation (dashed line), and those obtained with a simple approximation we will introduce in Appendix A. Noticing that the scale length over which $\Sigma_{\text {eff }}$ varies is given by the distance between entanglements $L_{e}$, our model predicts a very quick saturation, if compared with Brown's. 


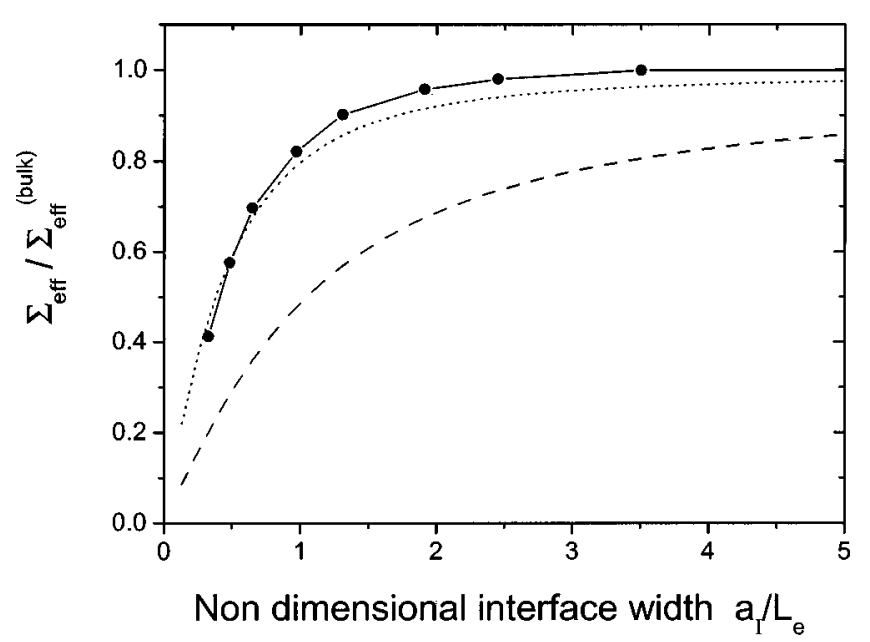

FIG. 2. Areal density of effective entanglements as a function of the interfacial width $a_{I}$, for the illustrative system described in the text. Joined circles are obtained from Eq. (20), dotted line from approximate Eq. (A6), and dashed line from Brown's formula (Ref. 6). $\Sigma_{\text {eff }}$ is scaled with respect to its bulk value $\Sigma_{\text {eff }}^{(\text {bulk }}=0.150 \mathrm{~nm}^{-2}$, and $a_{I}$ with respect to the entanglement length of the system $L_{e}=7.6 \mathrm{~nm}$.

In Fig. 3 variations of $\Sigma_{\text {eff }}$ with the molecular weight, for the same illustrative system, are shown, assuming $\chi=0.005$ constant and $N_{A}=N_{B}$. Increasing the molecular weight affects $\Sigma_{\text {eff }}$ in two opposite ways: through the interface width by lowering it and through chain end effects by increasing it. From the figure it is clear that chain end effects, that roughly contribute to $\Sigma_{\text {eff }}$ with a factor $1-2 M_{e} / M$, dominate, while the changes in width produce only a small correction. To demonstrate quantitatively that the chain end effects dominate, we fitted the calculated data with a function of the type $1-p M_{e} / M$, and obtained $p \approx 1.5$. The corresponding function is plotted in Fig. 3 as the solid line. This result is not surprising, since the dependence of the interfacial width on the molecular weight is weak. In fact, only close to miscibility can a large change in the width be obtained by varying the molecular weight, but we have seen in Fig. 2 that, for such large interfaces, $\Sigma_{\text {eff }}$ has already reached saturation.

Not many experimental data are available in the literature to validate our method, because it is difficult to devise a system in which interfacial width can be changed over a wide range of values, while keeping all other experimental conditions constant. The experimental possibilities are mainly three. It is possible to anneal two beams of the same material for different times, as it has been done for PS, ${ }^{4}$ but in this case the sample is not in thermodynamic equilibrium and it cannot be described by our method. It is also possible

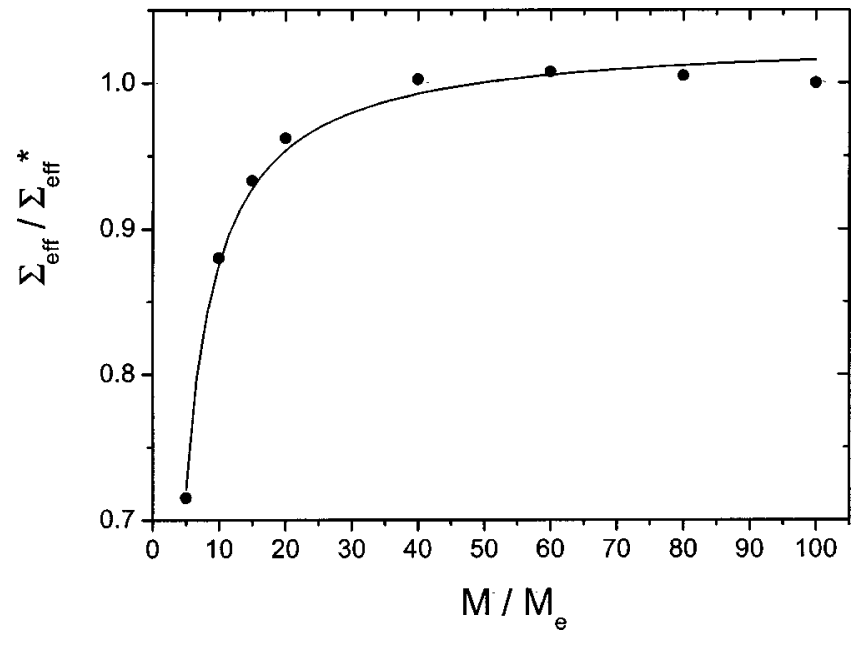

FIG. 3. Areal density of effective entanglements as a function of the molecular weight of the two polymers, calculated for the illustrative symmetric system described in the text with $\chi=0.005$ (full circles). Areal density is scaled with respect to $\Sigma_{\text {eff }}^{*}=0.134 \mathrm{~nm}^{-2}$, that is the value of $\Sigma_{\text {eff }}$ calculated for $M / M_{e}=100$. For comparison we plotted in solid line the function $\left(1-1.5 M_{e} / M\right)$.

to use two almost compatible materials and anneal them at different temperatures, as in the case of PS/PpMS. ${ }^{4}$ Finally, for strongly immiscible polymers, a wide range of interfacial widths can be obtained by using a random copolymer; interfaces PMMA/P(S-r-MMA) have been studied by Brown, ${ }^{6}$ $\mathrm{PS} / \mathrm{PBr}_{x} \mathrm{~S}$ by Schnell et al., ${ }^{5}$ and PS/PS-r-PVP by Benkoski et $a{ }^{19}{ }^{19}$ In this section we apply our model to two of the above systems, namely PMMA/P(S-r-MMA) and PS/PpMS.

Brown used a layer of a random copolymer P(S-r-MMA) placed between two sheets of PMMA. In a system like this it is possible to change the interface width by varying the PS fraction in the copolymer, while keeping the craze in PMMA. We decided to study this system because it explored a wide range of interfacial widths, compared with the entanglement lengths of the two materials. Furthermore, PS and PMMA are widely studied and there are many measurements available in the literature of their bulk properties. The parameters used in the calculations are reported in Table I.

The random copolymer is treated as a homopolymer with preaveraged parameters, and an empirical interaction parameter between PMMA and P(S-r-MMA) is introduced. This is simply an extension of Flory-Huggins theory and it is a widely used approach, that, even if it lacks a solid theoretical basis, could explain in many cases the enhanced miscibility of blends involving random copolymers. ${ }^{20,21}$ Since

TABLE I. Parameters used in the calculations. Other parameters: $f_{b}=1.115 \mathrm{nN}, U_{b}=5 \times 10^{-19} \mathrm{~J}$.

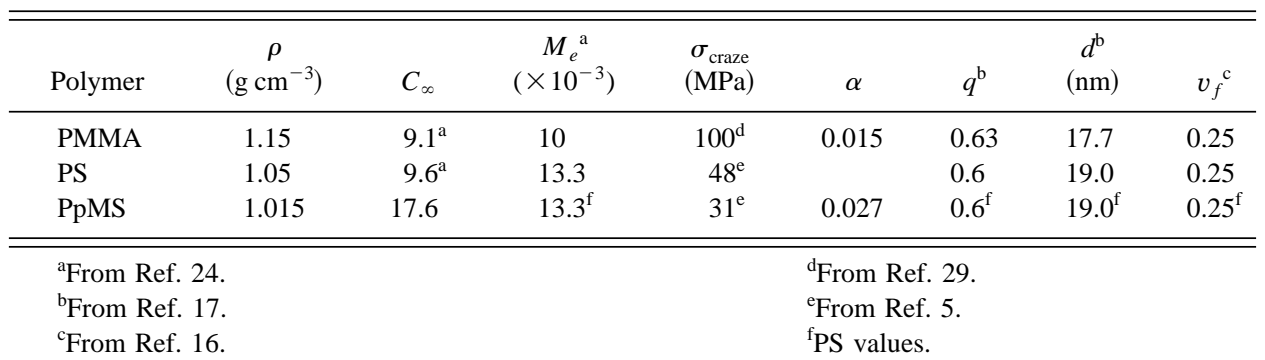




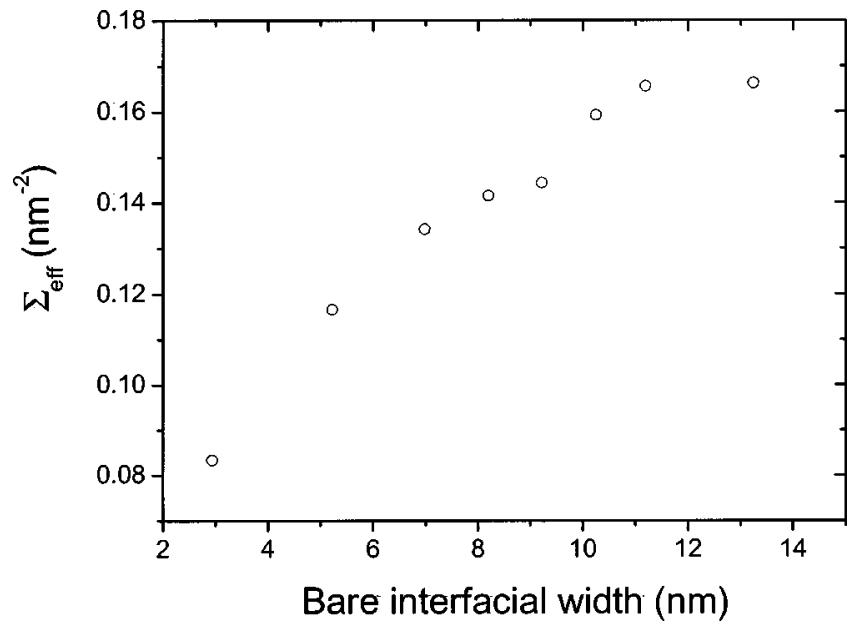

FIG. 4. Areal density of effective entanglements as a function of the bare interfacial width, calculated with our model [Eq. (20)] for the PMMA/P(Sr-MMA) samples investigated by Brown (Ref. 6).

we are working in a mean-field approximation, the same approach is suitable for our calculation of $\Sigma_{\text {eff }}$, provided that the copolymer is "ideal," meaning that all correlations are lost between the chemical identity of successive monomers. When it is possible the effective parameters for the copolymer are extracted from experimental results, but this is not the case and we have to choose appropriate interpolations. In our calculations, therefore, we assume that the corresponding homopolymer has the same degree of polymerization of the copolymer, and is made of identical monomers with $M_{0}^{\mathrm{h}}$ $=x M_{0}^{\mathrm{PS}}+(1-x) M_{0}^{\mathrm{PMMA}}$, where $x$ is the PS fraction in the copolymer. In a dense melt we can also assume that the mass density is given by the linear equation $\rho^{\mathrm{h}}=x \rho^{\mathrm{PS}}+(1$ $-x) \rho^{\mathrm{PMMA}}$. For the Kuhn segment length $b$, the most widely used approach is the Gaussian interpolation. It is assumed that the copolymer behaves as a Gaussian chain with two different segment lengths for the two species, so that its squared radius of gyration is $R_{g}^{2}=\left(x b_{\mathrm{PS}}^{2}+(1\right.$ $\left.-x) b_{\mathrm{PMMA}}^{2}\right) N / 6$. It follows that the correct expression for the Kuhn segment length of the equivalent homopolymer is $b_{\mathrm{h}}^{2}$ $=x b_{\mathrm{PS}}^{2}+(1-x) b_{\mathrm{PMMA}}^{2}$. The molecular weight of entanglement can be related to the above quantities using the packing model of Fetters et al. ${ }^{22}$ predicting

$$
M_{e} \propto \rho p^{3},
$$

where $p$ is the packing length

$$
p \propto \frac{M_{0}}{b^{2} \rho} .
$$

Considerable theoretical work has been done to derive an expression for the effective interaction parameter in blends involving random copolymers, ${ }^{23,24}$ but we prefer an empirical $\chi$ chosen so that the SCMF calculations would give experimental bare interfacial widths.

In Fig. 4 we report the calculated $\Sigma_{\text {eff }}$ as a function of the bare interfacial width for the PMMA/P(S-r-MMA) joints experimentally studied by Brown. ${ }^{6}$ The reason why the curve is not smooth is that in the experiments the molecular weight of the random copolymer was different for each sample.

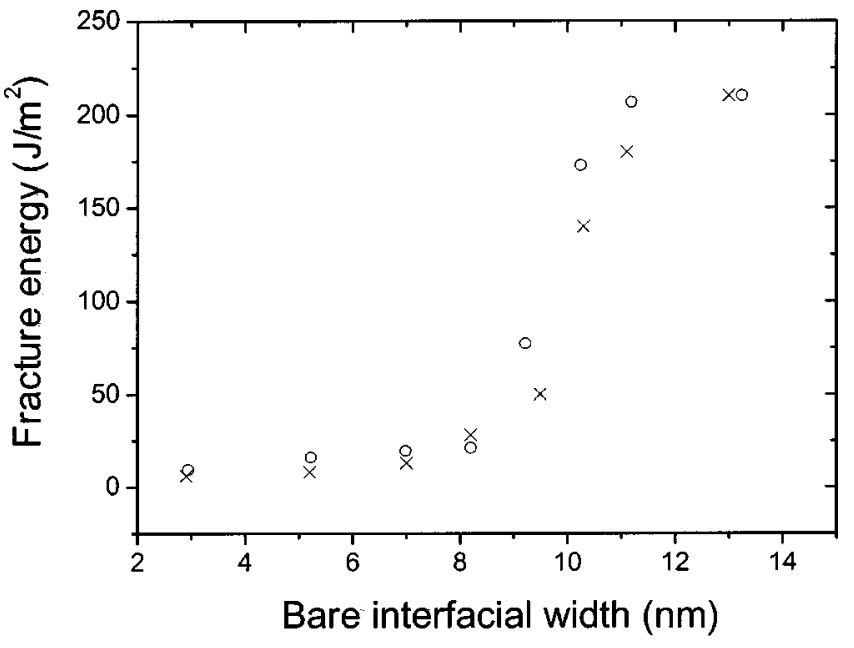

FIG. 5. Fracture energy as a function of the bare interfacial width for PMMA/P(S-r-MMA), calculated from the $\Sigma_{\text {eff }}$ given in Fig. 4 (circles). Crosses represent experimental data by Brown (Ref. 6).

The craze parameters for PMMA that we used in the fracture energy calculations are reported in Table I. The values for $\sigma_{\text {craze, }} v_{f}, d$, and $q$ have been found in the literature, while the other parameters have been chosen to fit the data. In particular, the value for $f_{b}$ was chosen such that it would give $\Sigma_{\mathrm{c}} / q \approx 0.142 \mathrm{~nm}^{-2}$, and correspondingly a transition to complete crazing for widths around $9 \mathrm{~nm}$, while $\alpha$ was chosen to fit the experimental fracture energy of the largest interface. The resulting fracture energy, calculated with our model, is compared with the experimental data by Brown ${ }^{6}$ in Fig. 5.

The fracture mechanisms predicted by the model are chain scission for the first pair, partial crazing for the following three samples, and complete crazing for the others. In the partial crazing regime we used $w=2 / 3$, that gives a critical length of about $120 \mathrm{~nm}$, at which the cross-tie fibrils start to transfer load. This last result is compatible with the analysis of Sha and co-workers, ${ }^{16}$ predicting that crazes with width less than three times the fibril length $(\approx 60 \mathrm{~nm})$ are not fully developed.

We notice that the agreement is good over the whole range of interfacial widths, agreement that is even more significant considering that we used literature values for most of the parameters.

The other system we studied is PS/PpMS. For PpMS we could find only the values of the density and of the crazing stress in the literature, while for most of the other parameters we simply used PS values, as shown in Table I. PpMS stiffness, $C_{\infty}$, was chosen to obtain the proper value for the Kuhn segment length of the blend, $b_{\mathrm{PS} / \mathrm{PpMS}}$ $=\sqrt{\left(b_{\mathrm{PS}}^{2}+b_{\mathrm{PpMS}}^{2}\right) / 2}=0.8 \mathrm{~nm}$, as found experimentally by Jung and Fisher. ${ }^{25}$ The interaction parameter $\chi=-0.011$ $+6.8 / T$ was chosen equal to that fitted by Schnell et al. ${ }^{4}$ and in agreement with the experimental results. ${ }^{25}$ Since $f_{b}$ was fixed by the previous fit, the only free parameter left is $\alpha$. Again, we used it to fit the highest experimental fracture energy. The results for $\Sigma_{\text {eff }}$ are displayed in Fig. 6 for the pairs PS $1.25 \mathrm{M} / \mathrm{PpMS}$ 570k and PS 139k/PpMS 157k. We 


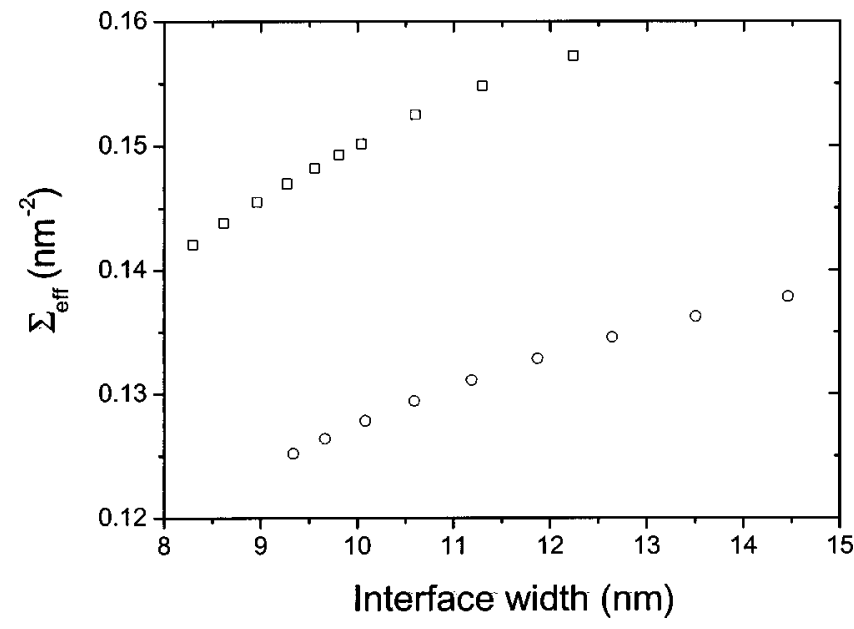

FIG. 6. Areal density of effective entanglements as a function of the bare interfacial width, calculated with our model [Eq. (20)] for two series of PS/PpMS interfaces: PS 1.25M/PpMS 570k (squares) and PS 139k/PpMS $157 \mathrm{k}$ (circles), at annealing temperatures ranging respectively from 120 to $210{ }^{\circ} \mathrm{C}$ and from 100 to $180{ }^{\circ} \mathrm{C}$.

note that for the investigated widths we are already in a saturation regime and that, fixing either width or temperature, the higher molecular weight sample shows the higher value of $\Sigma_{\text {eff }}$.

In Fig. 7 we show the corresponding calculated fracture energies, compared with the experimental data by Schnell et $a l .{ }^{4}$ Since in the experiments the interfacial width was not directly measured, we derived it by using the measured temperature and the fitted $\chi$ in the SCMF calculations; this is the reason why the experimental data in Fig. 7 look different from those in the original paper by Schnell and co-workers. ${ }^{4}$ Notice that even if the ratio of the predicted saturation values for the two molecular weights seems to be correct, the experimental data show a rapid increase of the fracture energy for the two samples, respectively, around 9 and $11 \mathrm{~nm}$, that is not reproduced by our model. As already discussed, the scale length over which saturation of $\Sigma_{\text {eff }}$ is reached, is given in

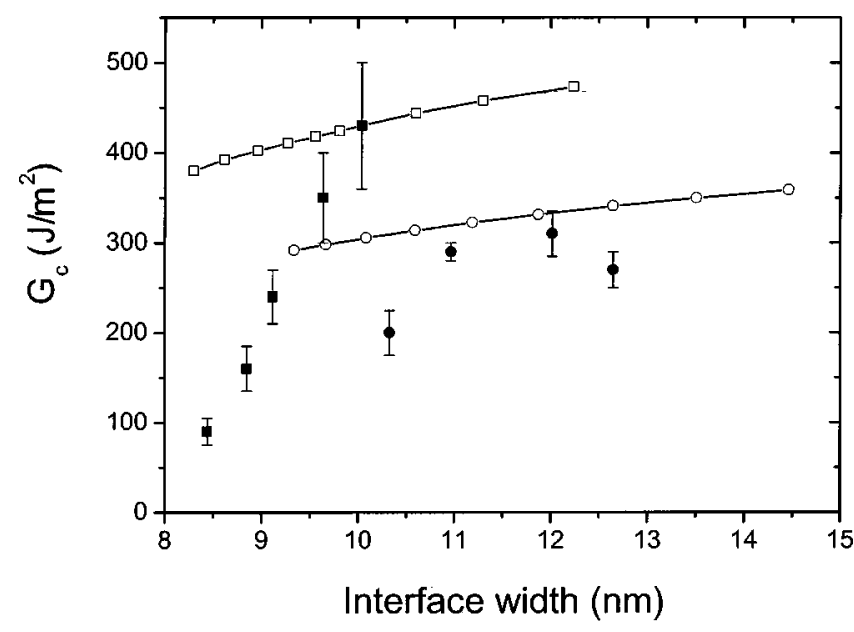

FIG. 7. Fracture energy of PS/PpMS interfaces, calculated from the $\Sigma_{\text {eff }}$ of Fig. 6 (joined empty symbols). Experimental data from Schnell et al. (Ref. 4) are also shown (full symbols). Two different pairs of molecular weights have been considered as in Fig. 6: PS 1.25M/PpMS 570k (squares) and PS 139k/PpMS 157k (circles). our model by the entanglements distance, that for this system is around $8 \mathrm{~nm}$; in the experiments, instead, fracture energy saturates much more quickly. Such a behavior of $G_{c}$ could be explained if the onset of crazing would arise for widths above $8.5 \mathrm{~nm}$, but using the set of parameters discussed above we predict a much smaller value for the critical width. In fact, for these samples crazing is the only predicted failure mechanism at all investigated temperatures. A more accurate estimate of PpMS parameters, would be needed to clarify this point.

Another possible explanation for the observed discrepancies between theory and experiments can be the fact that the molecular weight of entanglement at an interface is not the same as in the bulk. As already discussed by Brown and Russell, ${ }^{26}$ the entanglement density in the presence of an interface should be significantly reduced, leading to a smaller $\Sigma_{\text {eff }}$ for sharp interfaces than that predicted by our model. Ganesan and Pryamitsyn ${ }^{27}$ have described this effect quantitatively, and found that, for the systems they studied, an upper limit for $N_{e}$ at the interface is twice its bulk value. Future work will involve the inclusion of this correction in our model.

\section{CONCLUSIONS}

In this paper a new model to describe fracture of entangled asymmetric interfaces was proposed.

The core of the work is a new method to calculate the number of effectively entangled chain across an asymmetric interface, based on a rigorous mean-field approach, that has been widely used and tested in the past for dense polymer melts. An excellent approximate formula is also given in Appendix A for long chains, that allows a fast and simple application of the model.

Fracture energy has been calculated from $\Sigma_{\text {eff }}$ applying standard models for the mechanisms that we believe play a role in these systems. In addition, we also introduced a new regime called "partial crazing," that describes in a very simple way the fracture of an incomplete craze.

Theoretical predictions have been compared with experimental data for two different systems: the interface between PMMA and a random copolymer PS-r-PMMA, and the homopolymer blend PS/PpMS. For the former system the agreement was very good, while for the latter only the general trends could be reproduced. A possible explanation for the discrepancies has also been formulated.

In general, we believe that the limitations of the present work are mostly due to the scarce knowledge of the nature of the entanglement and this is the aspect that we are currently investigating.

\section{ACKNOWLEDGMENT}

The authors thank Robert Oslanec for helping with the numerical calculations and for letting them use his programs.

\section{APPENDIX A: LONG CHAINS APPROXIMATION}

In a $\nu$ chains system, the density of the $n$th monomers along the chains is given by 


$$
\rho_{n}(x)=\nu\left\langle\delta\left(x-x_{n}\right)\right\rangle=\frac{\rho_{b}}{N} q(x ; n) q(x ; N-n) .
$$

For chain end monomers the above formula reduces to

$$
\rho_{0}(x)=\rho_{N}(x)=\frac{\rho_{b}}{N} q(x ; N),
$$

as it is easily obtained by noticing that from definition (14) and Eq. (4) the boundary condition $q(x ; 0) \equiv 1$ follows.

In the two homogeneous phases far from the interface

$$
\frac{\rho_{0}( \pm \infty)}{\rho_{b}}=\frac{\rho_{N}( \pm \infty)}{\rho_{b}}=\frac{\phi( \pm \infty)}{N},
$$

from which follows $\phi( \pm \infty)=q( \pm \infty ; N)$.

In general, the density profiles $\rho_{n}(x)$ are not uniform and are a function of $n$, that indicates the position along the chain, through $q(x ; n)$. For infinite chains, we can neglect chain end effects and we can imagine that all the monomers are infinitely distant from the chain ends, so that $q(x ; n)$ will approach a function $q(x ; \infty)$ not depending on $n$. For the same reason all the monomers will have the same density distribution

$$
\frac{\rho_{n}(x)}{\rho_{b}} \equiv \frac{\phi(x)}{N},
$$

leading to

$$
q(x ; \infty)=\sqrt{\phi(x)} .
$$

Notice that approximation (A5) is the same used in the classical work of Helfand and Tagami. ${ }^{28}$

Substituting every $q(x ; n)$ with $q(x ; \infty)$ in Eq. (20) and considering a real $n_{e}=n-1$, we finally obtain

$$
\begin{aligned}
\Sigma_{\mathrm{eff}}= & \frac{2 \rho_{b}}{N}(n-2) \\
& \times\left[\int_{-\infty}^{0} d x \int_{0}^{\infty} d x^{\prime} \sqrt{\phi(x)} G_{0}\left(x, x^{\prime} ; N_{e}\right) \sqrt{\phi\left(x^{\prime}\right)}\right],
\end{aligned}
$$

where we also used $G_{0}$ as a rough approximation of the complete Green function. Expression (A6) is surprisingly good in approximating the accurate results for the entire range of interfacial width, as shown in Fig. 2. We also checked it for all the samples we studied, finding a comparable agreement.

\section{APPENDIX B: AN ALTERNATIVE POINT OF VIEW}

Expression (20) for $\left\langle\Sigma_{\text {eff }}\right\rangle$ can also be obtained in the following way. The number of effective entanglements is given by the mean density of $k$ th monomers times the probability that the following entanglement is on the other side of the interface. This can be represented as

$$
\Sigma_{\mathrm{eff}}=2 \sum_{k=1}^{n_{e}-1}\left[\int_{-\infty}^{0} d x_{i_{k}} \rho_{i_{k}}\left(x_{i_{k}}\right) \int_{0}^{\infty} d x_{i_{k+1}} p\left(x_{i_{k}}, x_{i_{k+1}} ; N_{e}\right)\right] \text {, }
$$

where symmetry of entanglement positions with respect to the chain center is used; see Eq. (15), and we denoted by $p\left(x_{n}, x_{m} ; m-n\right)$ the probability of finding the $m$ th monomer in $x_{m}$ given that the $n$th is in $x_{n}$. In terms of the Green function defined above, we have

$$
p\left(x_{n}, x_{m} ; m-n\right)=G\left(x_{n}, x_{m} ; m-n\right) \frac{q\left(x_{m} ; N-m\right)}{q\left(x_{n} ; N-n\right)},
$$

from which expression (20) can be recovered.

In the approximation of very long chains we can rewrite Eq. (A6) as

$$
\begin{aligned}
\Sigma_{\mathrm{eff}}= & \frac{2 \rho_{b}}{N}(n-2) \int_{-\infty}^{0} d x \phi(x) \\
& \times \int_{0}^{\infty} d x^{\prime} \sqrt{\frac{\phi\left(x^{\prime}\right)}{\phi(x)}} G_{0}\left(x, x^{\prime} ; N_{e}\right),
\end{aligned}
$$

and identify the expression in the second integral as an approximation for $p\left(x, x^{\prime} ; N_{e}\right)$.

In order to clarify the comparison with our model, and in particular with Eq. (B3), we also rewrite Brown's formula (1) as

$$
\Sigma_{\mathrm{eff}}=2 \rho_{e} \int_{-\infty}^{0} d x \phi(x) \int_{0}^{\infty} d x^{\prime} \frac{\phi\left(x^{\prime}\right)}{\phi(x)} \frac{\theta\left(L_{e}-\left|x^{\prime}-x\right|\right)}{2 L_{e}},
$$

where $\theta(x)$ is the usual step function. Recalling that

$$
\frac{2 \rho_{b}}{N}(n-2)=2 \rho_{e}\left(1-2 \frac{M_{e}}{M}\right),
$$

a comparison between the two expression is even clearer. In Brown's approach chain end effects are neglected, which is correct in the long chain limit, and chain connectivity is taken into account with a step function $\theta\left(L_{e}-\left|x^{\prime}-x\right|\right) / 2 L_{e}$ that approximates the usual Gaussian $G_{0}\left(x, x^{\prime} ; N_{e}\right)$. The main difference, producing the bigger discrepancies in the numerical results, is that for long chains the probability scales as $\sqrt{\phi\left(x^{\prime}\right) / \phi(x)}$, while in his model it is linear in volume fractions ratio.

${ }^{1}$ R. A. L. Jones and R. W. Richards, Polymers at Surfaces and Interfaces (Cambridge University Press, Cambridge, 1999).

${ }^{2}$ H. R. Brown, K. Char, V. R. Deline, and P. F. Green, Macromolecules 26, 4155 (1993).

${ }^{3}$ P. J. Cole, R. F. Cook, and C. W. Macosko, Macromolecules 36, 2808 (2003).

${ }^{4}$ R. Schnell, M. Stamm, and C. Creton, Macromolecules 31, 2284 (1998).

${ }^{5}$ R. Schnell, M. Stamm, and C. Creton, Macromolecules 32, 3420 (1999).

${ }^{6}$ H. R. Brown, Macromolecules 34, 3720 (2001).

${ }^{7}$ C. Creton, E. J. Kramer, C.-Y. Hui, and H. R. Brown, Macromolecules 25, 3075 (1992)

${ }^{8}$ C.-A. Dai, E. J. Kramer, J. Washiyama, and C.-Y. Hui, Macromolecules 29, 7536 (1996).

${ }^{9}$ E. J. Kramer, Adv. Polym. Sci. 52/53, 1 (1983).

${ }^{10}$ A. G. Mikos and N. A. Peppas, J. Chem. Phys. 88, 1337 (1988).

${ }^{11}$ P. G. de Gennes, C. R. Acad. Sci., Ser. II: Mec., Phys., Chim., Sci. Terre Univers 308, 1401 (1989).

${ }^{12}$ M. Doi and S. F. Edwards, The Theory of Polymer Dynamics (Clarendon, Oxford, 1986).

${ }^{13}$ J. Rottler, S. Barsky, and M. O. Robbins, Phys. Rev. Lett. 89, 148304 (2002).

${ }^{14}$ G. J. Lake and A. G. Thomas, Proc. R. Soc. London 300, 108 (1967).

${ }^{15}$ H. R. Brown, Macromolecules 24, 2752 (1991). 
${ }^{16}$ Y. Sha, C. Y. Hui, A. Ruina, and E. J. Kramer, Macromolecules 28, 2450 (1995).

${ }^{17}$ L. L. Berger, Macromolecules 23, 2926 (1990).

${ }^{18}$ K. R. Shull and E. J. Kramer, Macromolecules 23, 4769 (1990); K. R. Shull, J. Chem. Phys. 94, 5723 (1991).

${ }^{19}$ J. J. Benkoski, G. H. Fredrickson, and E. J. Kramer, J. Polym. Sci., Part B: Polym. Phys. 39, 2363 (2001).

${ }^{20}$ R. P. Kambour, J. T. Bendler, and R. C. Bopp, Macromolecules 16, 753 (1983).

${ }^{21}$ G. ten Brinke, F. E. Karasz, and W. J. MacKnight, Macromolecules 16, 1827 (1983).
${ }^{22}$ L. J. Fetters, D. J. Lohse, D. Richter, T. A. Witten, and A. Zirkerl, Macromolecules 27, 4639 (1994).

${ }^{23}$ G. H. Fredrickson and S. T. Milner, Phys. Rev. Lett. 67, 835 (1991).

${ }^{24}$ J. Dudowicz and K. F. Freed, Macromolecules 31, 5094 (1998).

${ }^{25}$ W. G. Jung and E. W. Fisher, Makromol. Chem., Macromol. Symp. 16, 281 (1988).

${ }^{26}$ H. R. Brown and T. P. Russell, Macromolecules 29, 798 (1996).

${ }^{27}$ V. Ganesan and V. Pryamitsyn, Macromolecules 35, 9219 (2002).

${ }^{28}$ E. Helfand and Y. Tagami, J. Chem. Phys. 56, 3592 (1972).

${ }^{29}$ W. Döll, Adv. Polym. Sci. 52/53, 105 (1983). 